\title{
Bacterial Hospital Acquired Infection in Port Sudan Teaching Hospital, Red Sea State, Sudan
}

\author{
Abd Elrahman Mustafa Abd Elrahman Osman*, Shingray Osman Hashim, \\ Mohammed Abdall Musa, Omer Mohammed Tahir
}

Medical Laboratory Sciences Division, Port Sudan Ahlia College, Port Sudan, Sudan

Email address:

abdo_rahman96@hotmail.com (A. E. M. A. E. Osman)

${ }^{*}$ Corresponding author

\section{To cite this article:}

Abd Elrahman Mustafa Abd Elrahman Osman, Shingray Osman Hashim, Mohammed Abdall Musa, Omer Mohammed Tahir. Bacterial Hospital Acquired Infection in Port Sudan Teaching Hospital, Red Sea State, Sudan. International Journal of Pharmacy and Chemistry. Vol. 4, No. 1, 2018, pp. 1-7. doi: 10.11648/j.ijpc.20180401.11

Received: December 2, 2017; Accepted: December 25, 2017; Published: January 15, 2018

\begin{abstract}
This study was carried out in Port Sudan teaching hospital from 8 November 2011 work to be initiated by the determination of level of aerobic bacterial contamination in the hospitals in port Sudan to 8November 2013. The aim of this study to isolate and identify different types of aerobic bacterial Hospital Acquired Infection, and determination of levels of antibiotic resistance of aerobic nosocomial isolate to commonly used antimicrobial agents. Two hundred samples collected from Urinary tract, Wound, Sputum, Ear, Throat, Blood, Personnel and different site of ward. The result showed that the isolated microorganism were as followed: Escherichia coli 33 (16.5\%), S. aureus 21 (10.5\%), Pseudomonas aeruginosa 20 (10\%), Klebsiella pneumonia 17 (8.5\%), Strepto Pyogene 9 (4.5\%), Strepto faecalis 7 (3.5\%), proteus mirabilis 4 (2\%), proteus vulgaris 4 (2\%), Morganella Morgenii 2 (1\%), Klebsiella oxytoca 2 (1\%), S. epidermidis 2 (1\%), Salmonella Para A 1 (0.5) Citrbacter Ferundii 1 (0.5\%), Serratia Spp $1(0.5 \%)$. Also the result showed that the majority of bacterial strains tested, exhibited marked most multiple drug resistance against some broad -spectrum antimicrobials used eg: Ceftazidime, it was evident that of the Gentamicin, Ciprofloxacin, Cefixime and Ceftriaxone is choice drug for Hospital Acquired Infection. This study concluded the effective control, prevention and treatment of infection. Knowledge of emerging pathogens and resistance profile is essential for treatment against Hospital Acquired infections.
\end{abstract}

Keywords: Hospital Acquired Infection, Urinary Tract Infections, Escherichia Coli, Port Sudan

\section{Introduction}

\subsection{Historical Background}

Since the use of temples by the Egyptians, attempts have been documented to provide special care protection and segregation for the thick. This collection of the thick has frequently resulted in rapid transmission of infectious agent among patients and personnel and from unrecognized reservoirs within the institution with resultant high morbidity and mortality [1]. The role of improved environmental sanitation and the importance of hygienic practice in reducing transmission and infection rates were recognized in the earliest medical traditions and have been periodically rediscovered in the following centuries. After Pasteur, the putrefaction associated with trauma and surgery was understood as related transmissible microorganism, and the importance of aseptic technique became will understood and practiced [2].

\subsection{Hospital-Acquired Infections}

Hospital-acquired infections characterized as any infection acquired in hospital. Community-acquired infections incubating on admission to hospital are excluded, though they may subsequently cause hospital infection in another patient. The principles are applicable to all health-care institutions including private medical practices. Recently, greater attention has been paid to the risks of hospitalacquired infections in staff as well as in patients [3].

\subsection{Impact of Nosocomial Infections}

Hospital-acquired infections add to functional disability 
and emotional stress of the patient and May, in some cases, leads to disabling conditions that reduce the quality of life. Nosocomial infections are also one of the leading causes of death. The economic costs are considerable $[4,5]$. The increased length of stay for infected patients is the greatest contributor to cost $[7,8,9]$. One study [6] showed that the overall increase in the duration of hospitalization for patients with surgical wound infections was 8.2 days, ranging from 3 days for gynecology to 9.9 for general surgery and 19.8 for ortho-pedicsurgery. Prolonged stay not only increases direct costs to patients or payers but also indirect costs due to lost work. The increased use of drugs, the need for isolation, and the use of additional laboratory and other diagnostic studies also contribute to costs. Hospital-acquired infections add to the imbalance between resource allocation for primary and secondary health care by diverting scarce funds to the management of potentially preventable conditions. The advancing age of patients admitted to health care settings, the greater prevalence of chronic diseases among admitted patients, and the increased use of diagnostic and therapeutic procedures which affect the host defenses will provide continuing pressure on nosocomial infections in the future $[7,8,9]$.

Organisms causing nosocomial infections can be transmitted to the community through discharged patients, staff, and visitors. If organisms are multi resistant, they may cause significant disease in the community [7, 8, 9].

\subsection{Factors Influencing the Development of Nosocomial Infections}

\subsubsection{The Microbial Agent}

The patient is exposed to a variety of microorganisms during hospitalization. Contact between the patient and a microorganism does not by itself necessarily result in the development of clinical disease other factors influence the nature and frequency of nosocomial infections. The likelihood of exposure leading to infection depends partly on the characteristics of the microorganisms, including resistance to antimicrobial agents, intrinsic virulence, and amount (inoculums) of infective material [1].

Many different bacteria, viruses, fungi and parasites may cause nosocomial infections. Infections may be caused by a microorganism acquired from another person in the hospital (cross-infection) or may be caused by the patient's own flora (endogenous infection). Some organisms may be acquired from an inanimate object or substances recently contaminated from another human source (environmental infection) [1].

Before the introduction of basic hygienic practices and antibiotics into medical practice, most hospital infections were due to pathogens of external origin (food borne and airborne diseases, gas gangrene, tetanus, etc.) or were caused by microorganisms not present in the normal flora of the patients (e.g. diphtheria, tuberculosis). Progress in the antibiotic treatment of bacterial infections has considerably reduced mortality from many infectious diseases. Most infections acquired in hospital today are caused by microorganisms which are common in the general population, in whom they cause no or milder disease than among hospital patients (Staphylococcus aureus, coagulasenegativestaphylococci, enterococci, Enterobacteriaceae) [1].

\subsubsection{Patient Susceptibility}

Important patient factors influencing acquisition of infection include age, immune status, underlying disease, and diagnostic and therapeutic interventions. The extremes of life infancy and old age are associated with a decreased resistance to infection. Patients with chronic disease such as malignant tumors, leukemia, diabetes mellitus, renal failure, or the acquired immunodeficiency syndrome (AIDS) have an increased susceptibility to infections with opportunistic pathogens. The latter are infections with organism (s) that are normally innocuous, e.g. part of the normal bacterial flora in the human, but may become pathogenic when the body's immunological defenses are compromised. Immunosuppressive drugs or irradiation may lower resistance to infection. Injuries to skin or mucous membranes bypass natural defense mechanisms. Malnutrition is also a risk. Many modern diagnostic and therapeutic, such as biopsies, endoscopic examinations, catheterization, intubation / ventilation and suction and surgical procedures increase the risk of infection. Contaminated objects or substances may be introduced directly into tissues or normally sterile sites such as the urinary tract and the lower respiratory tract [1].

\subsubsection{Environmental Factors}

Health care settings are an environment where both infected persons and persons at increased risk of infection congregate. Patients with infections or carriers of pathogenic microorganisms admitted to hospital are potential sources of infection for patients and staff. Patients who become infected in the hospital are a further source of infection. Crowded conditions within the hospital, frequent transfers of patients from one unit to another, and concentration of patients highly susceptible to infection in one area (e.g. newborn infants, burn patients, and intensive care) all contribute to the development of nosocomial infections. Microbial flora may contaminate objects, devices, and materials which subsequently contact susceptible body sites of patients. In addition, new infections associated with bacteria such as waterborne bacteria (atypical mycobacterium) and/or viruses and parasites continue to be identified [7, 8, 9].

\subsubsection{Bacterial Resistance}

Many patients receive antimicrobial drugs. Through selection and exchange of genetic resistance elements, antibiotics promote the emergence of multi-drug resistant strains of bacteria; microorganisms in the normal human flora sensitive to the given drug are suppressed, while resistant strains persist and may become endemic in the hospital. The widespread use of antimicrobials for therapy or prophylaxis (including topical) is the major determinant of resistance. Antimicrobial agents are, in some cases, becoming less effective because of resistance. As an antimicrobial agent becomes widely used, bacteria resistant to this drug eventually 
emerge and may spread in the health care setting. Many strains of pneumococci, staphylococci, enterococci, and tuberculosis are currently resistant to most or all antimicrobials which were once effective. Multi-resistant Klebsiella and Pseudomonas aeruginosa are prevalent in many hospitals. This problem is particularly critical in developing countries where more expensive second-line antibiotics may not be available or affordable $[7,8,9]$.

\subsection{Classification of Hospital-Acquired Infections}

i. Reservoirs and sources of infection.

ii. Routes of transmission.

iii. Rupture of our host defenses.

Iv. Resultant major infections.

\subsubsection{Reservoirs and Sources of Infection}

Infectious sources are either endogenous (from the patient's own normal flora) or exogenous. The latter derive from people (hospital staff or another patient), usually directly, or from the environment in hospital, usually indirect spread from sources such as air, dust, linen, food, water and other fluids (including disinfectants, and intravenous fluids) or equipment such as endoscopes and ventilators. Some of these can be a reservoir (e.g., infected fluid) which contaminates sources (bottles or bowls), so eradication of the source is insufficient and the reservoir must also be found [3].

\subsubsection{Routes of Transmission}

Spread of infection can occur by: direct contact; person-toperson cross-infection, usually via the hands (sexually transmitted infection is rare). Indirect contact; via some object, as above. Common vehicle: a special form of indirect contact in which one infected vehicle, commonly food or fluid, infects many. Airborne spread: either by droplets travelling 1-2 meters, or smaller droplet nuclei travelling a kilometer or more. Vector borne spread: unusual but not unknown in hospitals) [3].

\subsubsection{Rupture of Our Host Defenses}

The portals of entry are the urogenital tract, skin, respiratory tract and the mouth and the gastrointestinal tract. High-risk patients have impaired host defenses form: age; the very young and the elderly. Immune defects from disease: e.g. HIV, diabetes, hepatic or renal impairment, cancer or lymphoma, especially with neutropenia. Immune defects from drugs: e.g. cytotoxic, steroids or immunosuppressive (in transplant patients). Immune antibody defects: lack of vaccination or previous exposure (chicken-pox, CMV, hepatitis B). Organ defects: e.g. pre-existing urinary, lung or skin disease. Skin and tissue defects: trauma, surgery and treatment; multi-trauma and /or multi-access results in multi-risk [3].

\subsubsection{Resultant Major Infections}

The major four types of infection are: urinary tract infections (about 40\%). Surgical wound infections (about 25\%). Lower Respiratory Infections (about 10). Bacteremia (about 5\%). Skin ulcer, pressure sore, gut and other infections make up the remaining 20\% [3].

\subsection{Bacterial Causative Organisms}

The major pathogens are S. aureus, E. coli and other enteric Gram-negative rods and P. aeruginosa, though their relative importance differs in the major infections Less important pathogens are Enterococci, coagulase-negative staphylococci, anaerobes and Candida spp. Multi-resistance is common now and important [3].

\section{Materials and Method}

\subsection{Study Approach}

Study approach is to identify and isolate the possible causative organisms and the characterization of isolated strains using bio typing and antibiograms.

\subsubsection{Study Type and Design}

Cross sectional descriptive study.

\subsubsection{Study Area}

The study area are Include (different department of Port Sudan Teaching Hospital), Red Sea Corporation and some Private Hospitals in Port Sudan city on Red Sea state eastern Sudan.

\subsubsection{Study Period}

This was cross-sectional study Conducted during the period from Augest2011 to Augest2013.

\subsubsection{Study Population}

All patients admitted at different hospital unit and set in hospital more than $48 \mathrm{hs}$, were monitored daily by attending physicians for subsequent development of H. A. I.

\subsection{Methodology}

\subsubsection{Sample Size and Data Collection}

According to standard method Two Hundred specimens were collected and examined to detect, and identify pathogens or their products using: microscopic examination of specimen to detect their motility, morphology and staining reaction. Also culture techniques to isolate pathogens in pure form and to identify them, then do biochemical reactions to identify pathogens by their enzymatic and fermentative reactions and then test their antibiotic sensitivity test. (Major Socio-economic Indicators for Population. Khartoum 2003) [10]. (Red Sea State Center for Health Information. Report of year 2004.) [11].

\subsubsection{Sampling}

Samples for Hospital Acquired Infections to investigate and diagnosis of microbial diseases involves (urine specimen, Sputum, swabs (wound, ear, throat), Blood and other specimens) (Major Socio-economic Indicators for Population. Khartoum 2003) [13], (Red Sea State Center for Health Information. Report of year 2004.) [11]. 


\subsubsection{Cultivation of Specimens}

All specimens were inoculated onto appropriate medium

\subsubsection{Identification of the Isolated Bacteria}

Colonial morphology, direct and indirect gram stain, Biochemical tests and Antimicrobial sensitivity test we do to all isolated organisms.

\subsubsection{Statistical Data Processing}

Laboratory results will be statistically using a Statistical product and Service Solutions (SPSS) program.

\section{Results}

Two hundred specimens include (50 urine specimens, 50 wound swab, 20 Ear swab, 15 Throat swab, 25 Sputum, 10 Blood and 30 other specimens). Were collected from patients Suspected Hospital Acquired Infection through the study period from August 2011 to August 2013. Out of 200 patients 124 (62\%) were positive, while $76(38 \%)$ were negative, Among the positive growth $34(17 \%)$ of them were NLF organisms, and $81(40.5 \%)$ were L. F organisms while 9 (4.5\%) organism's dose not grow on Macconkey agar but shown significant growth with characters of beta hemolysis on Blood agar. The Gram reaction show that the Gram negative most frequent $85(42.5 \%)$, Gram positive less frequent $39(19.5 \%)$.

The patients constitute $101(50.5 \%)$ female and $89(44.5 \%)$ male. The age of the patients ranged from less than 12 years to more than 12years. Age more than 12 years are most frequent $181(90.5 \%)$, while age less than 12 year rare less frequent $9(4.5 \%)$.

The isolated Microorganism were as followed: Escherichia coli 33 (16.5\%), S. aureus 21 (10.5\%), Pseudomonas aeruginosa 20 (10\%), Klebsiella pneumonia 17 (8.5\%), Strepto Pyogene 9 (4.5\%), Strepto faecalis 7 (3.5\%),, proteus mirabilis $4(2 \%), \%)$, proteus vulgaris $4(2 \%)$, Morganella Morgenii 2 (1\%), Klebsiella oxytoca 2 (1\%), S. epidermidis 2 (1\%), Salmonella Para A 1 (0.5) Citrbacter Ferundii 1 (0.5\%), Serratia Spp 1 (0.5\%).

Out of 50 urine specimens $34(68 \%)$ were shown positive culture while $16(32 \%)$ shown negative culture, The isolated organisms from urine specimen revealed as followed: E. coli 20 (40\%), Klebsiella pneumonia $3(6 \%)$, Strepto faecalis 3 (6\%), Klebsiella Oxytoca 2 (4\%), Morganella morgenii 2 (4\%), proteus mirabilis 1 (2\%), Proteus vulgaris 1 (2\%), Citrbacter ferundii 1 (2\%) salmonella Para A 1 (2\%).

The gender study show that the most frequent patients were female $36(72 \%), 23(46 \%)$ of them are shown positive culture, male are less frequent $14(28 \%), 11(22 \%)$ of them shown positive culture.

Out of 50wound swabs specimen 42 (84\%) shown positive culture while $8(16 \%)$ shown negative culture. And the isolated organisms included Pseudomonas aeruginosa 13 (26\%), E. coli 8 (16\%), S. aureus 8 (16\%), Klebsiella Pneumonia 5 (10\%), proteus vulgaris 3 (6\%), St. Faecalis 3 (6\%), proteus mirabilis 2 (4\%).
Out of 25 sputum specimens, 7 (28\%) were shown positive culture while $18(72 \%)$ shown negative culture. And the isolated organisms revealed as followed: Klebsiella pneumonia 6 (24\%), Serratia spp 1 (4\%).

From 20 Ear swab specimen $12(60 \%)$ were positive while $8(40 \%)$ were negative. And the isolated organisms included pseudomonas aeruginosa $4(20 \%)$, S. aureus $4(20 \%)$, Klebsiella pneumonia 2 (10\%), proteus vulgaris 1 (5\%), E. coli $1(5 \%)$.

Out of 15 throat swab specimen $9(60 \%)$ were shown positive culture while $6(40 \%)$ shown negative culture. The only isolated organism is Strepto pyogene.

Out of 10 Blood specimen $4(40 \%)$ were shown positive culture while $6(60 \%)$ shown negative culture. And the isolated organisms are E. coli $1(10 \%)$, Klebsiella pneumonia $1(10 \%)$, pseudomonasauruginosa $1(10 \%)$, Strepto faecalis 1 $(10 \%)$.

Out of 30other specimens $16(53.3 \%)$ shown positive culture while $14(46.7 \%)$ were shown negative culture. The isolated organisms revealed as followed: S. aureus $6(20 \%)$, E. coli 4 (13.3\%), Pseudomonas aeruginosa 2 (6.7\%), S. epidermidis 2 (6.7\%), Klebsiella pneumonia 1 (3.3\%), proteus mirabilis 1 (3.3\%) (figure 1$)$.

All isolated organisms were subjected to antimicrobial sensitivity test using Kirby-Bauer disc diffusion method. Testing sensitivity of isolates to spectrum of antibiotic is a commonly used epidemiological tool. Standardized KirbyBauer disk gel diffusion testing provide useful guidance both to the clinician for appropriate therapy and to the epidemiologist as an in expensive screen for possible common identity of suspected organisms [1] Table 1.

Table 1. Pattern of Antimicrobial sensitivity and resistant for each Antibiotic used.

\begin{tabular}{lllllll}
\hline \multirow{2}{*}{ No } & \multirow{2}{*}{ Antimicrobial } & \multicolumn{2}{c}{ Sensitive } & \multicolumn{2}{c}{ Resistant } \\
\cline { 3 - 7 } & & Disc Potency & No & \% & No & \% \\
\hline 1 & Amoxicillin & $10 \mathrm{mcg}$ & 20 & 10 & 104 & 52 \\
2 & Ciprofloxacin & $5 \mathrm{mcg}$ & 97 & 48.5 & 27 & 13.5 \\
3 & Cefixime & $5 \mathrm{mcg}$ & 94 & 48.2 & 25 & 12.8 \\
4 & Ceftazidime & $30 \mathrm{mcg}$ & 88 & 45.1 & 30 & 15.3 \\
5 & Ceftriaxone & $30 \mathrm{mcg}$ & 93 & 47.8 & 25 & 12.9 \\
6 & Co. trimoxazole & $25 \mathrm{mcg}$ & 67 & 34.4 & 52 & 26.8 \\
7 & Clindamycin & $2 \mathrm{mcg}$ & 29 & 14.5 & 12 & 6 \\
8 & Gentamicin & $10 \mathrm{mcg}$ & 110 & 55 & 14 & 7 \\
9 & Nitrofurantoin & $300 \mathrm{mcg}$ & 43 & 21.5 & 60 & 30 \\
10 & Nalidixic acid & $30 \mathrm{mcg}$ & 50 & 25 & 53 & 26.5 \\
11 & Amikacin & $10 \mathrm{mcg}$ & 84 & 42 & 3 & 1.5 \\
12 & Ofloxacin & $5 \mathrm{mcg}$ & 83 & 41.5 & 12 & 6 \\
13 & Tetracycline & $30 \mathrm{mcg}$ & 60 & 30 & 72 & 36 \\
14 & Erythromycin & $15 \mathrm{mcg}$ & 18 & 9 & 3 & 1.5 \\
15 & Vancomycin & $30 \mathrm{mcg}$ & 23 & 11.5 & 0 & 0 \\
16 & Amoxyclav & $30 \mathrm{mcg}$ & 36 & 18 & 12 & 6 \\
\hline & & & & & & \\
\hline
\end{tabular}




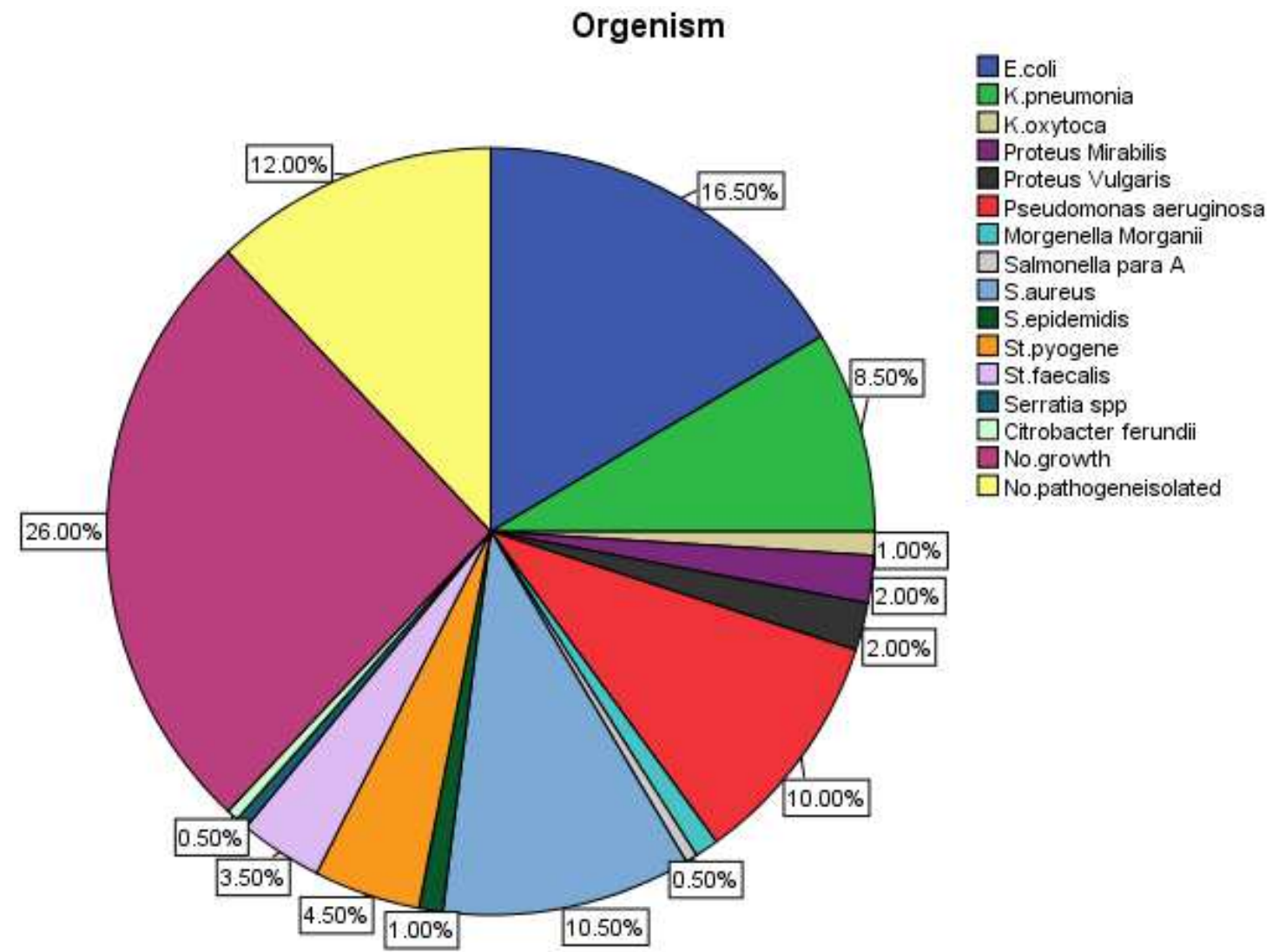

Figure 1. Percentage of each isolated organism.

\section{Discussion}

Hospital-acquired infections have increased worldwide, contributing considerably to morbidity and mortality of the hospitalized patients [12]. Therefore, prolonging hospital stay can add significantly to the economic burden of underlying disease. Hospitalized patients acquire nosocomial infections due to important risk factors including advancing age, intravenous lines, indwelling urinary catheter and surgical wounds in intensive care units (ICU) and/or medical specialties wards. In this study we tried to evaluate and detect the prevalence of aerobic bacteria responsible for nosocomial infections in patients suffering from (urinary tract infections among hospitalized patients at Port Sudan Teaching Hospital, Red Sea State, Sudan [12].

Out of 50, $34(68 \%)$ patients had developed urinary tract infections during study period. urinary tract infections is the most common Hospital Acquired infection, and studies suggest that patients with such infections have an increased institutional death rate and this result agreed with the result obtained by [13]. Study added, In our study bacteria were isolated from patients in 3 days up to 7 days, thus agreeing with the previous study in the maximum number of 7 days [13]. Hospital Acquired urinary tract infections are the largest institutional reservoir of nosocomial antibiotic-resistant pathogens that agree with results obtained by [14]. The results revealed that the lactose ferment bacterial types represent $(60 \%)$, while non-lactose fermented organisms represent (12\%) and this result agreed with the result obtained by. The ratio between male to female about $1: 2$, this result is similar to result report by [15]. The result showed that UTI cases among female of age group more than 12 years were found more susceptible to urinary tract infection, that agree with results obtained by [15].

The study revealed that elder males more than 12 years are more susceptible to urinary tract infection this result agree with a result obtained by [16].

In the present study the most prevalent organisms were $\mathrm{E}$. coli $(40 \%)$ of all urine clinical specimens, this result nearly to the result obtained by [17]., who showed that $E$. coli account $(62 \%)$, the followed organisms are Klebsiella pneumonia (6\%), Strepto faecalis (6\%), Klebsiella Oxytoca (4\%), Morganella morgenii (4\%), proteus mirabilis (2\%), Proteus vulgaris (2\%), Citrbacter ferundii (2\%) and salmonella Para A (2\%) respectively.

In previous studies in the United States Acute-care hospitals reported E. coli (26\%), K. pneumonia (12\%), P. mirabilis $(6 \%), P$. vulgaris $(10 \%)$ and $P$. aeruginosa $(16 \%)$, [18]. Another supporting research to this study was that conducted in Saudi Arabia to evaluate the prevalence of gram-negative bacteria implicated in urinary tract infections due to Foley's urinary catheter. The research showed that $30.3 \%$ of E. coli isolated, which is higher than reported figures of E. coli in Canada (2.7-6.2\%) and USA (2.2-6.6\%). The increasing antimicrobial resistance among bacteria 
causing urinary tract infections makes therapy of this type of infections difficult and leads to more use of extensive broadspectrum drugs. In our study, antimicrobial susceptibility of bacterial isolates from urinary tract infections showed different patterns but the most susceptible antibiotic is Gentamicin with higher percentage (34\%) and the lowest susceptible is Amoxicillin (16\%).

Gram-negative bacteria were also frequently responsible for wound infections in a study conducted in New Orleans, USA, among these organisms were P. mirabilis, P. vulgaris, E. coli and Ps. aeruginosa. This study also looked at the susceptibility patterns of bacteria responsible for wound infections.

The reported incidence of nosocomial pneumonia (NP) in varies across different studies, which may be explained by the presence of different populations with variable ages and other associated risk factors. Incidence ranges from $6.8 \%$ to $27 \%$ [19] and this agreed our study $7 \%$ associated risk factor. As Gram-negative bacteria are documented to be the most common causative agents of NP [20] and this agreed our study.

Furthermore, In previous study pneumonia comprises approximately one-third of nosocomial infections in hospitals of Saudi Arabia. To reduce the incidence of NP, it is important to take into consideration the risk factors for NP that can be managed, and all those involved in hospital management need to set practical and effective guidelines for prevention of Hospital acquired infection. In our study out of $25,7(28 \%)$ had developed nosocomial pneumonia and this is higher with the previous study in different Egypt hospital. The present study revealed that the $K$. pneumoniae were the major causes of HAI Pneumonia infections (24\%). while other bacteria isolated is Serratia spp (4\%).

In the present study, out of $10,4(40 \%)$ represent the nosocomial blood stream infection (BSI). These results were higher than those reported by [18] in Canada. The highest number of cases was reported form the coronary ICU (43/159 patients), which nearly our study.

In the present study we also tried to evaluate and detect the prevalence of aerobic bacteria responsible for nosocomial infections in patients suffering from (Ear and Throat infections). Ear and Throat infections is the one of common Hospital Acquired infection,. In our study, the prevalence of Ear infections among the recruits for the study period, out of 20 ear specimens $60 \%$ had developed Ear Infection. Furthermore, the most prevalent organisms were $P$. aeruginosa $20 \%$ followed by $S$. aureus $20 \%$, K. pneumonia $10 \%$, P. vulgaris $5 \%$ and E. coli $5 \%$. The ratio between male to female is $2: 1$, The result showed that Ear infection cases among female of age group (more than 12 years) were found more susceptible to Ear infection (95\%), also the gram negative is common isolated organisms in ear infection and this result were agreed the previous study carried by Emerging infection disease in united state. In the Throat infection out of $15,9(60 \%)$ patients had developed throat Infection. In the present study the only isolated organism is st. pyogene, patient more than 12 years are more susceptible to infection, the result obtained in Ear and Throat infection is slightly higher $10.5 \%$ than the result obtained by W. H. O in Ear Nose and Throat infections 7\%. Also in our study the physician, medical laboratory staff, nurse, Worker, lotion, gauge, cotton, patient bed, floor, door handle, beside bed and equipment were investigated to identified the source of infection.

\section{Conclusion and Recommendation}

\subsection{Conclusion}

Hospital Acquired infections are associated with a great deal of morbidity, mortality and increased financial burden. Intensive care is a risk factor for the emergence of antibiotic resistant bacteria. Gram-negative bacteria have overtaken Gram-positive organisms as the predominant cause of Hospital Acquired infections. Inadequate antibiotic therapy is associated with poor outcome and particularly with bacterial resistance. Infection control measures are important for the effective control, prevention and treatment of infection. Knowledge of emerging pathogens and resistance profile is essential for treatment against Hospital Acquired infections. Shorter duration of treatment and correct dosage of antibiotic therapy is recommended to reduce the selection pressure for resistant isolates. Hand washing is the single most important measure to prevent Hospital Acquired infections. Gloves must not be used as a substitute for hand washing; they must be washed on glove removal.

Now a day multiple drug resistant has become, commonly observed in strains of pathogenic bacteria.

The widely application and misuse of antimicrobial agents markedly increased the occurrence of multiples -drug resistance in microorganisms and there with limits the use of most available and affordable therapeutic agents. The most prevalent organism in hospital acquired infections is E. coli.

Also the most prevalent organism in hospital acquired urinary tract infection is E. coli. And the most prevalent organism in hospital acquired wound infection is pseudomonas aeruginosa, the most prevalent organism in hospital acquired ear infection is Pseudomonas aeruginosa and $S$. aureus. Also the most prevalent organism in hospital acquired throat infection is Strepto pyogene, the most prevalent organism in hospital acquired pneumonia is Klebsiella pneumonia.

Female are more susceptible to UTI, Pneumonia, Ear and throat infection than male. But male are more susceptible to wound infection and Septicemia than female. Ages more than 12 years are the most infected. Gentamicin is highest active agents in most patient with H. A Urinary Tract Infections. Cefixime is highest active agents in most patient with $\mathrm{H}$. A Pneumonia. Cefixime and Erythromycin are the highest active agents in most patient with $\mathrm{H}$. A Throat Infections. Gentamicin is the highest active agents in most patient with H. A Ear Infections. Cefixime is highest active agents in most Patient with H. A septicemia. Gentamicin is highest active agents in H. A wound infection. Amoxicillin is the 
lowest active antimicrobial agents due to high resistant rate.

\subsection{Recommendations}

I. Screenings test should be done to detect carrier among hospital staff.

II. Increase awareness about the hazards of using antibiotic for treatment without pre -therapy culture and how bacteria gain resistant among people.

III. Establishment of antimicrobial policies and treatment guidelines.

IV. Uses of Gentamicin as routine treatment for hospital acquired U. T. I and Ear infection.

V. Uses of Cefixim as routine treatment for hospital acquired pneumonia, septicemia and throat infection.

VI. Uses of Amikacin, Gentamicin, Ciprofloxacin and Ofloxacin as routine treatment for hospital acquired wound infection.

VII. Decontamination of hospital environments.

VIII. Do further study to detect the degree of sterilization in operation room.

IX. Do further study to detect prevalence of hospital acquired viruses.

X. Uses of API $20 \mathrm{E}$ are recommended for investigation of all Enterobacteriaceae in Microbiology lab for more accuracy.

XI. In depth studies by using modified technique (molecular methods) for precision and accuracy is recommended.

\section{References}

[1] Evans, A. E; and Feldmen, H. A., (1982). In: Bacterial Infection of Humans, Epidemology and Control. A. S Evans and H. A. Feldman (eds), Plenum Pub. Co., New York, pp. 367-368 and 371-372.

[2] Toledo-Pereyra, LH.; and Toledo, M. M., (1976). A critical study of Lister's work onantiseptic surgery. A m J. Surg., 131: 736-744.

[3] Spicer. W. J, clinical Bacteriology, Mycology and Parasitology. CHURCHILL LIVING STONE: 2000: Page 190-192.

[4] Greenwood, Slack. Richard. C. B and Pentherer, John. F. (2002). Medical publishers Microbiology, A guide to microbial infection, sixteen edition, P. 250-275. Churchill living stone.

[5] Gupta K. Emerging antibiotic resistance in urinary tract pathogens Infect Dis Clin North Am. 2003; 17 (2): 243-59.

[6] Mims Cedric, dock ell. Hazel. M, Goering. V, Riot. Ivan,
Wakelin. Derek and. Zuckerman. Mark. (2004). Medical Microbiology, third edition, ELSEVIER MOSBY.

[7] Haussen Karen. (2005). Infection Control Staff Fact Sheet, Infection control Unit, Women's and Children's Hospital, Children, Youth and Women's Health Service, Government of South Australia.

[8] Hooton TM. Clinical practice. Uncomplicated urinary tract infection. N Engl J Med 2012; 366 (11): 1028-1037.

[9] Jawetz, Mel nick and Adel berg (2007). Medical Microbiology 24th edition, McGraw-Hill companies.

[10] Movahedian AH, Mosayebi Z, Moniri R. Urinary Tract Infections in Hospitalized Newborns in Beheshti Hospital, Iran: A Retrospective Study. J Infect Dis Antimicrob Agents. 2007; 24 (1): 7-11.

[11] EL-MISHAD ABLAM. Manual of Practical Microbiology. Eighth Edition. Cairo: Kalyoub-Egypt, 2006: 149-157.

[12] Janković S, Mirović V, Marković-Denić L, Romić P, Mikić D: Nosocomial bloodstream infections in ICU and non-ICU patients. Am J Infect Control 2005, 33: 333-40.

[13] Daschner F, Chiarello LA, Dettenkofer M, Fabry J, Francioli $\mathrm{P}$, Knopf $\mathrm{H}-\mathrm{J}$, et al. Hygiene and infection control of nosocomial catheter-associated urinary tract infection. In: Naber KG, Pechere JC, Kumazawa J, et al., editors. Nosocomial and health care associated infections in urology. London: Health Publication Ltd; 2001. pp. 105-119.

[14] Janković S, Mirović V, Marković-Denić L, Romić P, Mikić D: Nosocomial bloodstream infections in ICU and non-ICU patients.

[15] Orret and Shurland, 1998. Bacterial etiologic agents of urinry tract infection in Hospitalized patients Nigeria, Abuja.

[16] Torkaman M, Afsharpaiman SH, Hoseini MJ, Moradi M, Mazraati A, Amirsalari S, et al. Platelet count and neonatal sepsis: a high prevalence of Enterobacter spp. Singapore Med J. 2009; 50 (5): 482-5.

[17] Eriksen HM, Iversen BG, Aavitsland P. Prevalence of nosocomial infections in hospitals in Norway, 2002 and 2003. J Hosp Infect. 2005; 60: 40-5.

[18] Sharifian M, Karimi A, Tabatabaei SR, Anvaripour N. Microbial sensitivity pattern in urinary tract infections in children: a single center experience of 1,177 urine cultures. Jpn J Infect Dis. 2006; 59 (6): 380-2.

[19] Pitout. Johann. D. D, Nordmann, Laupland. Kevin. B and Poirel. (2005). Emergence of Enterobactericeae Producing Extended Spectrum B-lactamase (ESBLs) in community, Journal of Antibacterial Chemotherapy, 56 (1): 52-59.

[20] Wang JT, Chang SC, Chen YC, Luh KT. "Comparison of antimicrobial susceptibility of Citrobacter freundii isolates in two different time periods." The Journal of Microbiology, Immunology and Infection. 2000 Dec; 33 (4): 258-62. 